\title{
Size Dependent Fractal Aggregation Mediated through Surfactant in Silica Nanoparticle Solution
}

\author{
Sugam Kumar ${ }^{1, *}$, V.K. Aswal ${ }^{1}$ and J. Kohlbrecher ${ }^{2}$ \\ ${ }^{I}$ Solid State Physics Division, Bhabha Atomic Research Centre, Trombay, Mumbai 400 085, INDIA \\ ${ }^{2}$ Laboratory for Neutron Scattering, Paul Scherrer Institute, CH-5232 PSI Villigen, Switzerland \\ "E-mail: sugam@barc.gov.in
}

\begin{abstract}
Small-angle neutron scattering (SANS) has been used to study aggregation of anionic silica nanoparticles in presence of cationic surfactant (DTAB) in aqueous solution. The measurements were carried out for different sizes of nanoparticles $(8.2,16.4$ and $26.4 \mathrm{~nm})$ at fixed $(1 \mathrm{wt} \%)$ nanoparticles and surfactant concentration. It is found that the adsorption of surfactant micelles on the silica nanoparticles leads to the aggregation of nanoparticles, which is characterized by a fractal structure. The number of adsorbed micelles on nanoparticle increases from 7 to 152 with the increase in the size of the nanoparticle from 8.2 to $26.4 \mathrm{~nm}$, whereas interestingly the fractal dimension remains same. The aggregate morphology in these systems is expected to be governed by the diffusion limited aggregation.
\end{abstract}

Keywords: Silica nanoparticle, cationic surfactant, fractal aggregate, Self-assembly, SANS.

PACS: 81.07.-b, 82.70.Uv, 83.85.Hf, 87.15. nr

\section{INTRODUCTION}

Aggregation of nanoparticles is of scientific interest because of its multitude applications in the area of molecular biology, food processing, electro-optics etc [1]. In general, aqueous colloids attain long term stability through electrostatic or steric repulsion. However, in the presence selective additives, several attractive forces such as hydrophobic/hydrophilic interactions, the formation of coordination or covalent bonds and the interaction of complimentary functional or biological groups may lead to the aggregation phenomenon [2]. The presence of an electrolyte usually leads to coagulation (liquid-solid transition) whereas the presence of oppositely charged macromolecules such as surfactant and protein gives rise to the liquid-liquid phase transition characterized by fractal structure. Surfactant self-assemble to from micelles in aqueous solution and their adsorption on nanoparticle governs the particle aggregation. In the present work, we have examined the size dependence of the nanoparticle on micelle adsorption and corresponding changes in their resultant structures using SANS. Fractals are self-similar objects and in the case of mass fractal the distribution of mass varies with a power $D$ of length scale $R$ smaller than the space dimension. Small-angle neutron scattering (SANS) with its unique advantage of contrast variation is useful technique for characterizing such multicomponent systems [3]. In SANS, the intensity $I(Q)$ for fractals is proportional to a negative power of wave vector transfer $Q$ as given by $I(Q) \sim Q^{-D}$. Further, the adsorption of micelles on the nanoparticles can be directly observed by selectively contrast matching the required component.

\section{EXPERIMENTAL}

Electrostatically stabilized colloidal suspensions of three sizes of silica nanoparticles (Ludox SM30, HS40 and TM40) and cationic surfactant DTAB (Dodecyltrimethyl ammonium bromide) were obtained from Sigma-Aldrich. Samples were prepared by dissolving $1 \mathrm{wt} \%$ amount of silica nanoparticles and surfactants in $\mathrm{D}_{2} \mathrm{O}$. Some of the samples were also prepared in contrast-matched solvent for DTAB surfactant. Small-angle neutron scattering experiments were performed at SANS-I facility, Swiss Spallation Neutron Source SINQ, Paul Scherrer Institut, Switzerland. Data were collected at two sample-todetector distances 2 and $8 \mathrm{~m}$ to cover a $Q$ range of 0.008 to $0.3 \AA^{-1}$. Corrections were made for background and empty cell contributions and data were normalized to absolute cross-sectional unit using standard procedure.

\section{SANS ANALYSIS}

In SANS, one measures the coherent scattering intensity as a function of $Q$ which can be given by [4] 


$$
I(Q) \sim n_{a} V^{2} \rho_{p}-\rho_{s}{ }^{2} P(Q) S(Q)+B
$$

where $n_{a}$ is the number density of particles and $V$ is particle volume. $\rho_{p}$ and $\rho_{\mathrm{s}}$ are scattering length densities of particle and solvent, respectively. $P(Q)$ is intraparticle structure factor and $S(Q)$ is interparticle structure factor. $P(Q)$ has been calculated using coreshell structure of the micelles adsorbed on the nanoparticle and $S(Q)$ for particle aggregation modeled by a mass fractal. $B$ is a constant term representing incoherent background.

\section{RESULTS AND DISCUSSION}

Fig. 1 shows the SANS data of $1 \mathrm{wt} \%$ DTAB with $1 \mathrm{wt} \%$ HS40 silica nanoparticles along with respective pure components. The scattering data of silica particles shows a monotonically decreasing function governed by $P(Q)$ as $S(Q) \sim 1.0$. On the other hand, a correlation peak is observed for DTAB because of peak in $S(Q)$ [3]. The scattering data from mixed system is significantly different from simple addition of the data of pure components (inset of Fig. 1). The data clearly show a high scattering with linear dependence in the low Q region indicating the formation of fractal structure particle aggregates in the system [4]. The scattering at high $Q$ region matches to that from the DTAB micelles which could be either adsorbed on nanoparticle or free in the mixed system.

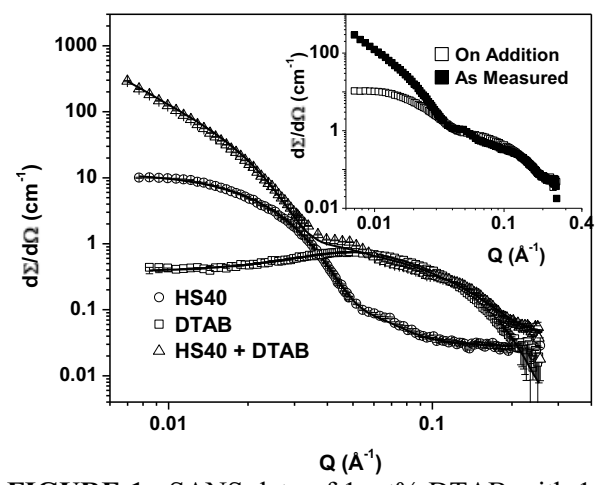

FIGURE 1. SANS data of $1 \mathrm{wt} \%$ DTAB with $1 \mathrm{wt} \%$ HS40 silica nanoparticles along with respective pure components. Inset shows the comparison of addition of data for pure components with that experimentally measured.

The SANS data for $1 \mathrm{wt} \%$ DTAB contrast-matched to the solvent with $1 \mathrm{wt} \%$ of nanoparticles SM30 $(8.2 \mathrm{~nm})$, HS40 $(16.4 \mathrm{~nm})$ and TM40 $(26.4 \mathrm{~nm})$ are depicted in Fig. 2. In this case, the scattering is only from fractal aggregates of silica nanoparticles. The fitted parameters are listed in Table 1. It is interesting to note that irrespective the size of the nanoparticle the fractal dimension is same. The SANS data for the same systems without contrast-matched are shown in the Inset of the Fig. 2. The scattering corresponds to contributions from both fractal aggregates and micelles.

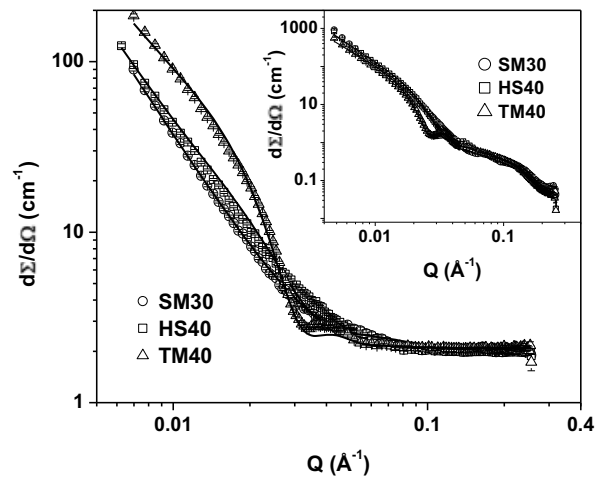

FIGURE 2. SANS data for with $1 \mathrm{wt} \%$ DTAB contrastmatched to the solvent with $1 \mathrm{wt} \%$ of nanoparticles SM30, HS40 and TM50. Inset shows the data for the same systems when no component is contrast-matched.

TABLE 1. Fitted parameters for silica nanoparticles and DTAB complexes.

\begin{tabular}{ccccc}
\hline $\begin{array}{c}\text { Nano- } \\
\text { particles }\end{array}$ & $\begin{array}{c}\text { Particle } \\
\text { size } \\
(\AA)\end{array}$ & $\begin{array}{c}\text { Fractal } \\
\text { dimension } \\
(\mathrm{D})\end{array}$ & $\begin{array}{c}\text { Adsorbed } \\
\text { micelles } \\
\text { per } \\
\text { particle }\end{array}$ & $\begin{array}{c}\text { Vol \% of } \\
\text { adsorbed } \\
\text { micelles }\end{array}$ \\
\hline SM30 & 41 & 2.2 & 7 & 44 \\
HS40 & 82 & 2.3 & 34 & 26 \\
TM40 & 132 & 2.3 & 152 & 23 \\
\hline
\end{tabular}

It is found that the number of micelles adsorbed on nanoparticle increases from 7 to 152 with increasing particle size from 8.2 to $26.4 \mathrm{~nm}$. On the other hand, the overall adsorption (vol\%) of micelles is significantly reduced with the increase in the size of the nanoparticles as expected because of decrease in the total surface area for the larger particles at fixed $\mathrm{wt} \%$ concentration. We have found that particle aggregation in the present system has the same structure $(\mathrm{D} \sim 2.3)$ irrespective of the nanoparticle size. This constant value of fractal dimension may be attributed to the site specific aggregation of particles mediated through the adsorbed of micelles. A fractal dimension 2.3 indicates a diffusion limited aggregate (DLA) type of fractal morphology. These fractals are usually formed when the density of particles is quite low and the repulsive forces are relatively weak as is the present case.

\section{REFERENCES}

1. R. Mezzenga et al, Nature Materials 4, 729 (2005)

2. D. Li and R. B. Kaner, J. Am. Chem. Soc. 128, 968 (2006)

3. Sugam Kumar and V. K. Aswal J. Phys.: Condens. Matter 23035101 (2011).

4. Sugam Kumar, V.K. Aswal and J. Kohlbrecher, Langmuir 27, 10167 (2011)

5. T.A Witten and L.M Sander, Phys. Rev. Lett. 47 14001403 (1981). 